\title{
Modeling resistive switching in nanogranular metal films
}

\author{
Walter Tarantino ${ }^{*}$ and Luciano Colombo $\odot$ \\ Dipartimento di Fisica, Università degli Studi di Cagliari, Cittadella Universitaria, I-09042 Monserrato, Cagliari, Italy
}

(Received 10 July 2020; accepted 3 December 2020; published 18 December 2020)

\begin{abstract}
Films produced by assembling bare gold clusters well beyond the electrical percolation threshold show a resistive switching behavior whose investigation has started only recently. Here we address the challenge to charaterize the resistance of a nanogranular film starting from limited information on the structure at the microscopic scale by the means of Bruggeman's approach to multicomponent media, within the framework of effective medium approximations. The approach is used to build a model that proves that the observed resistive switching can be explained by thermally regulated local structural rearrangements.
\end{abstract}

DOI: 10.1103/PhysRevResearch.2.043389

\section{INTRODUCTION}

The synthesis of materials and devices with new properties by means of controlled manipulation of their structure down to the atomic scale is nowadays paving the way to a plethora of new technological applications. Among these, neuromorphic computing architectures [1], which are believed to have the potential of overcoming the looming end of Moore's scaling law, are becoming possible thanks to "resistive switching junctions" [2], that is, devices that suddenly change their resistance under the action of an electric current in a nonvolatile and reversible way. Resistive switching (RS) behavior has been reported in several systems, from oxides to semiconductors to organic materials [3,4]. In particular, networks of nanowires, nanoparticles and clusters can also be used to make RS devices. This can be achieved by creating insulator-conductor nanocomposites, namely by embedding the metallic components into a polymeric matrix or passivating them by a shell of ligands or oxide layers [3,5]. Purely metallic nanoparticle systems can also present RS behavior if randomly deposited on a substrate to form a highly discontinuous film near the percolation threshold [6-8]. In all those systems, RS emerges as an insulator-to-metal transition due to the formation of a conducting percolation network $[3,9]$, which can be described in terms of percolation theory [10-12].

Recently, cluster-assembled gold films have been reported to present an unexpected form of resistive switching $[13,14]$. By supersonic cluster beam deposition of bare Au nanoparticles on various substrates, metallic films characterized by a complex microstructure have been grown well beyond their electrical percolation threshold. Nanoparticles

\footnotetext{
*walter.tarantino@dsf.unica.it

Published by the American Physical Society under the terms of the Creative Commons Attribution 4.0 International license. Further distribution of this work must maintain attribution to the author(s) and the published article's title, journal citation, and DOI.
}

deposited on the substrate basically retain their individuality, thus forming films of (nano)granular matter (henceforth "ng-films"), a medium highly porous and rich of interfaces among misoriented crystal grains. Besides increasing the electrical resistance of ordinary (i.e., continuous or, equivalently, atom-assembled) thin films, their nanostructure results in a remarkable dynamical response to a sufficiently high applied voltage. Even under the action of a direct electric current a ng-film presents a resistance characterized by nearly ohmic regimes (during which only small fluctuations occur) and abrupt jumps to higher as well as to lower values, after which the system either returns to the same ohmic regime (spikes) or reaches a new one (steps). As the system is well above its electrical percolation threshold, the underlying mechanisms differ from the usual insulator-to-metal transition responsible for RS in other systems. Therefore, they need to be elucidated by means of further experimental as well as theoretical investigations and this work represents an attempt to build some appropriate theoretical tools for such an endeavor.

The outcome of a complete theoretical characterization of such a RS phenomenon would be a model able to reproduce the abrupt changes in resistance and to provide a reasonable estimate of the spanned values. The challenge is twofold. On the one hand, one needs to identify and characterize the underlying mechanisms responsible for the jumps of resistance. On the other hand, one must be able to estimate the resistance of a ng-film, which, even in the ohmic regime, is not at all an easy task to accomplish, as the highly inhomogeneous nature of the system is not captured by the standard models used to estimate the resistance of crystal films. The aim of the present work is therefore to establish a theoretical framework that allows to estimate the resistance of a ng-film starting from ingredients which could be accessible to ab initio and semiempirical methods. In other words, the present attempt to elaborate an adequate formalism linking the observed RS behavior to some underlying microstructure evolution should pave the way to a multiphysics approach, where the resulting theoretical model will be fed by physical information computed from first principles. 
The first steps in that direction were made already in Ref. [13], where the experimental results were qualitatively reproduced using a dynamical resistor network model. The approach is very flexible and has been used to explain many emergent properties of nanoparticle and nanowire networks [9,15-22]. However, the computational cost of solving the Kirchhoff's equations implied by a nanoscale resolved effective resistor network modeling a macroscopic threedimensional sample can be very demanding. As we are interested in a regime far from the percolation threshold, we do not need to rely on such a precise description and we can resort to other, approximate and thus more affordable, methods. We therefore adopt an effective medium approximation (EMA), which in principle allows to estimate the electrical resistivity of a ng-film starting just from limited knowledge about its microscopic structure [23-25]. In Sec. II we present the approach and explain how, in a steady situation, it can be used to account for the high degree of porosity of a structure, as well as for other structural features that define the nanogranular character of the system. In Sec. III we add a dynamical evolution due to thermally regulated structural changes, and show that they indeed rule over the observed RS behavior; we also explain how they can be modeled within our framework. Two realizations of this modeling are shown to capture many features of the experimentally reported ng-film resistance [13,14].

Such an analysis allows us to trace a clear path for future investigations aimed at identifying the actual atomic-scale mechanisms occurring in the films. Full extent and limits of the approach are discussed in the last section.

\section{OHMIC REGIME}

\section{A. Synopsis of the residual resistivity contributions}

Before addressing the dynamical evolution of a ng-film, we must discuss its conductance in a static regime. Since its dynamical response is only obtained for high-enough applied voltages, the static regime can be taken as the ohmic regime occurring when a weak induced current is used as a probe. For stronger currents, the response is describes as nearly ohmic regimes punctuated by jumps of resistance. This, together with the observation that during the occurrence of the RS phenomenon the film does not seem to undergo any qualitative structural change at the macroscopic scale, allows us to hypothesize that the inhomogeneities characterizing the ohmic regime at the microscopic scale remain qualitatively the same also during the dynamical regime. In other words, the sources of electronic scattering characterizing the ohmic regime we are about to discuss remain the same during the dynamical one. What changes is their quantitative contribution to the overall resistance of the sample.

Taking apart the contribution of the temperature, which, as we will see, we believe to play an important role in the dynamical evolution, we focus for now on the residual resistivity (RR) of a ng-film. It is known that for such systems the RR can be orders of magnitude higher than that of films of the same metal in the crystalline phase [26]. In fact, the $\mathrm{RR}$ of these latter can already differ quite sensibly from that of the bulk material. Grain boundaries and surface effects are known to play a major role [27] and the models of MayadasShatzkes [28] and Fuchs-Sondheimer [29,30] are routinely used to account for them. In the case of ng-films, additional structural imperfections have a higher impact on the overall $\mathrm{RR}$. We organize such imperfections in the following way. Foremost we consider the contribution of voids, namely the interstitial vacuum existing among nanoparticles. Although they do not allow for band transport, they still allow hopping and tunneling, whose contribution to the overall current is, however, expected to be much lower than band transport. A second relevant source of RR are the interfaces between nanoparticles. In the best case, they can be assimilated to boundaries between randomly oriented grains belonging to different nanoparticles. Most likely, however, an amorphous layer is present in the contact region, as a result of the impact during the deposition stage [31]. To best of our knowledge, there is no well-established method in literature to account for neither imperfections in estimating the resistivity of a film.

Surface effects, which may become relevant for thin films, can be estimated using the Fuchs-Namba model [32], an extension of Fuchs formalism [29] that also takes into account the roughness of the top layer of nanoparticles. The presence of grains, on the other hand, is not adequately described by the model of Mayadas-Shatzkes, which assumes that grains grow in a columnar structure, with the axes normal to the film plane, and extend from two surfaces of the film. While usually valid for continuous crystal films, these assumptions are not justified for nanogranular ones, where grains are randomly oriented, as a result of the nanoparticle deposition, and therefore do not satisfy the assumptions of the model. Impurities and further defects inside the grains add the last contribution to the RR.

Such a hierarchy of contributions to the RR of a nanonagranular film is summarized in Fig. 1. Since the RR determined by the imperfections pictorially depicted in Figs. 1(a), 1(e) and 1(f) can be accurately estimated by means of wellestablished theoretical tools, in the following we shall focus on the modeling of Figs. 1(b), 1(c) and 1(d).

\section{B. EMA approach}

In the study of RS devices, electrical transport properties of inhomogeneous media are generally studied within the framework of percolation theory [3], a statistical approach to the study of the formation of a percolation network [11,12]. RS indeed typically emerges as a consequence of an insulator-toconductor transition of a composite material, occurring when the concentration of the conducting component crosses the "percolation threshold." Far from that threshold, as the case we are interested in, the conductivity of an inhomogeneous medium can also be accurately estimated by means of an EMA $[23,33,34]$. Within such an approach, the inhomogeneous medium is effectively treated as a homogeneous one whose properties are calculated in a mean-field approximation. For regimes well above the percolation threshold, such simplification allows to accurately describe very complex systems with simple analytical formulas [10,12,35]. Among all possible realizations of the approach, we adopt here Bruggeman's one $[23,36]$, which allows to deal with a mixture of several components treating them all on the same footing 


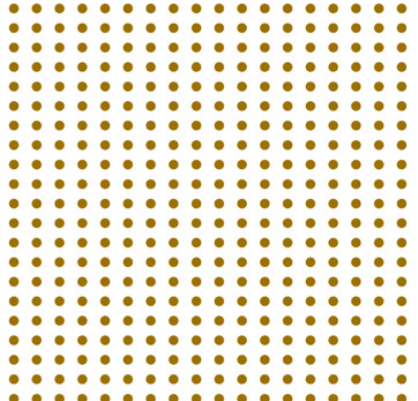

(a)

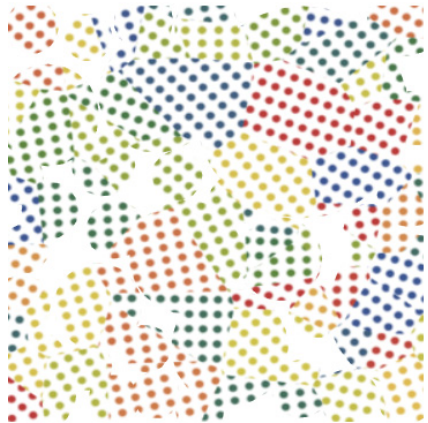

(c)

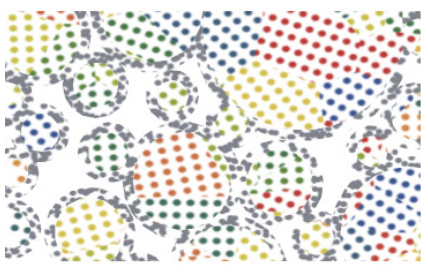

(e)

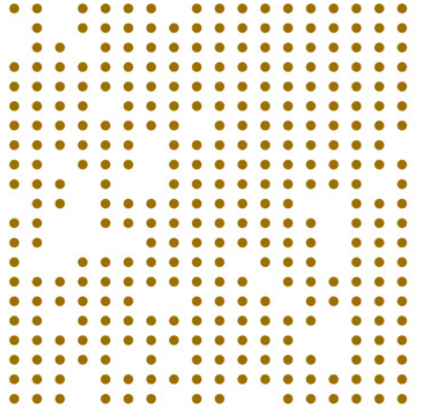

(b)

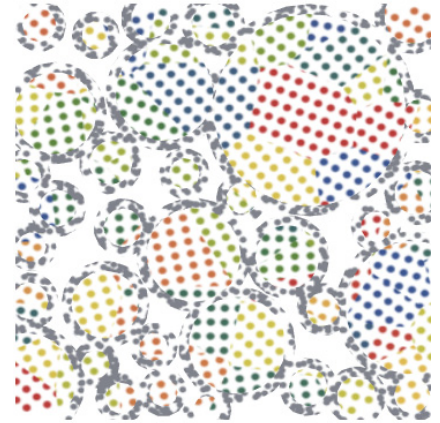

(d)

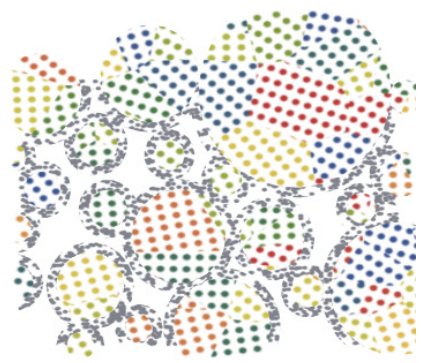

(f)
FIG. 1. The RR of a ng-film can be regarded as arising from the contribution of a variety of sources of electronic scattering: (a) the crystalline structure, (b) the interstitial voids, (c) the presence of randomly oriented grains (denoted by different colors), (d) the presence of thick nanoparticle interfaces, (e) surface effects due to the "thinness" of the film, and (f) roughness of the surface, impurities, and other defects.

(see Fig. 2). Such an approach offers a natural framework for dealing with the different inhomogeneities enumerated in the previous section, as we shall explain.

Given an inhomogeneous system made of a certain number of homogeneous components, the conductivity of the system is estimated via the "effective conductivity" $\sigma_{e}$ calculated as the positive root of the equation

$$
\sum_{i} \Phi_{i} \frac{\sigma_{i}-\sigma_{e}}{\sigma_{i}+2 \sigma_{e}}=0
$$

where $i$ runs over all the components, $\Phi_{i}$ is the relative volume fraction occupied by the component $i$, for which $\sum_{i} \Phi_{i}=1$, and $\sigma_{i}$ is the corresponding conductivity. Such a formula concerns an arbitrary number of conducting components mixed in the inhomogenous system. An insulating component can

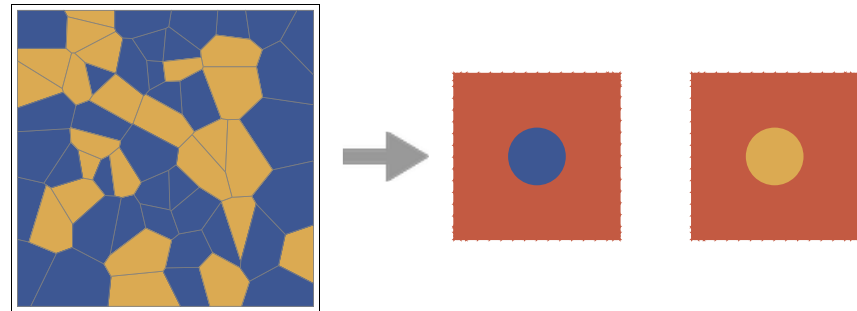

FIG. 2. A pictorial representation of Bruggeman's formalism. On the left, the real system, which is composed by a mixture of two components characterized by their electrical conductivity. On the right, the model, in which a single region (of either components) is assumed to be a sphere embedded in a single medium of uniform conductivity. As an external field is turned on, the presence of the sphere causes a certain polarization. By imposing that the average polarization due to all regions cancels out, one deduces the relation Eq. (1), which allows to determine the conductivity of the medium $\sigma_{e}$ in a self-consistent manner [23].

be also considered by simply using a vanishing conductivity. Indeed, a first, crude estimate the resistivity of a ng-film can be obtained by considering the film as a mixture of bulk gold, for which $\sigma_{\mathrm{Au}}=0.041 \mathrm{n} \Omega^{-1} \mathrm{~m}^{-1}$ at room temperature [37], and insulating vacuum, $\sigma_{V}=0$. Assuming that the nanoparticle density in the film is close to that of a set of randomly packed spheres, we consider $\Phi_{\mathrm{Au}} \approx 0.63$ [38], and hence $\Phi_{V}=1-\Phi_{\mathrm{Au}} \approx 0.37$, in Eq. (1) to estimate the resistivity of a ng-film to be $\rho \approx 55 \mathrm{mn} \Omega$, which is twice as much that of bulk gold. A more accurate estimate can be obtained if we improve the description of the gold component. For instance, we can include the effects of grain boundaries by taking its conductivity to be that of a polycrystalline film with similar grain size. Using $\sigma_{\mathrm{Au}} \approx 0.01 \mathrm{n} \Omega^{-1} \mathrm{~m}^{-1}$, which is the conductivity of a film with grains of approximately $10 \mathrm{~nm}$ (see Ref. [39] Table 1, sample S1), the resistivity of our ng-film is estimated to be $\rho \approx 225 \mathrm{mn} \Omega$. This value, which is about 10 times that of a crystalline film, is comparable with the values reported by Mirigliano et al. of $\rho \sim 100 / 1000 \mathrm{mn} \Omega$ [13], which suggests that our approach is sound.

The flexibility of the EMA approach, in fact, allows us to be even more precise. Suppose that within the highly inhomogeneous metal component we can identify regions of relatively uniform conductivity, which we say belong to the same "phase": Equation (1) can be accordingly reinterpreted as referring not to a mixture of just two components (vacuum and metal), but to a mixture of different phases, which can also belong to the same component (in our case, the metal one). The classification of the various inhomogeneities of a ng-film exposed in the previous section helps us in identifying phases in our systems. For instance, if we believe that amorphous layers at the interfaces between nanoparticles account for an important contribution to the total resistivity, then one can single that contribution out by introducing three phases: a vacuum, an amorphous, and a polycrystalline phase. If, on the other hand, one believes that nanoparticle interfaces do not contribute more than simple grain boundaries, but the size of nanoparticles is more important, then one can introduce different phases to describe nanoparticles of different size. For instance, in Ref. [13] it is reported 
TABLE I. Resistivity estimates $(\rho)$ for static four-phase ng-film, for different values of phase concentrations $\left(\Phi_{i}\right)$ and relative conductivities $\left(\sigma_{i} / \sigma_{\mathrm{Au}}\right.$, with $\left.\sigma_{\mathrm{Au}}=0.041 \mathrm{n} \Omega^{-1} \mathrm{~m}^{-1}\right)$, keeping fixed the vacuum concentration and conductivity to $35 \%$ and 0 , respectively.

\begin{tabular}{lllcccc}
\hline \hline$\sigma_{1} / \sigma_{\mathrm{Au}}$ & $\sigma_{2} / \sigma_{\mathrm{Au}}$ & $\sigma_{3} / \sigma_{\mathrm{Au}}$ & $\Phi_{1}$ & $\Phi_{2}$ & $\Phi_{3}$ & $\rho$ \\
\hline 0.122 & 0.0244 & 0.0122 & $26 \%$ & $26 \%$ & $13 \%$ & $1173 \mathrm{~m} \mathrm{n} \Omega$ \\
0.122 & 0.0244 & 0.0122 & $32.5 \%$ & $32.5 \%$ & $0 \%$ & $950 \mathrm{mn} \Omega$ \\
0.122 & 0.0244 & 0.0122 & $52 \%$ & $13 \%$ & $0 \%$ & $566 \mathrm{~m} \mathrm{n} \Omega$ \\
0.122 & 0.0244 & 0.0122 & $13 \%$ & $52 \%$ & $0 \%$ & $1581 \mathrm{~m} \mathrm{n} \Omega$ \\
1.000 & 0.0244 & 0.0122 & $26 \%$ & $26 \%$ & $13 \%$ & $509 \mathrm{~m} \mathrm{n} \Omega$ \\
0.488 & 0.244 & 0.0122 & $26 \%$ & $26 \%$ & $13 \%$ & $180 \mathrm{~m} \mathrm{n} \Omega$ \\
\hline \hline
\end{tabular}

that two distinct nanoparticle populations characterized by a different radius $(0.7$ and $4.4 \mathrm{~nm})$ are clearly discernible, at least in the initial part of the deposition. One can therefore introduce a phase for the population of smaller nanoparticles and one for the larger ones. A resistivity of about $\rho \sim 1000$ $\mathrm{mn} \Omega$ is obtained, for instance, by considering four phases: one for the vacuum, for which $\sigma_{0}=0$ and $\Phi_{0}=0.35$, a first metallic phase, which might represent large nanoparticles, with $\sigma_{1}=0.005 \mathrm{n} \Omega^{-1} \mathrm{~m}^{-1}$ and $\Phi_{1}=0.26$, a second, lessconducting, metallic phase, representing small nanoparticles, with $\sigma_{2}=0.001 \mathrm{n} \Omega^{-1} \mathrm{~m}^{-1}$ and $\Phi_{2}=0.26$, and a third, even less-conducting, metallic phase, representing amorphous layers between the nanoparticles, with $\sigma_{3}=0.0005 \mathrm{n} \Omega^{-1} \mathrm{~m}^{-1}$ and $\Phi_{3}=0.13$. The precise values for these quantities must be inferred either from further experimental measurements or atomistic simulations. For actual samples, they can presumably span a wide range of values. To get an idea of how much the resistivity estimate varies in different situations, in Table I we report estimates for a variety of situations.

\section{MODELING DYNAMICAL PROCESSES}

The discussion so far assumed that the system is in a static condition, characterized by a ohmic response to an applied direct current. In experiments, for a sufficiently strong applied current, ohmic regimes are interrupted by RS phenomena, which must reflect some sort of microscopical structural change of the system. Due to the high degree of inhomogeneity of ng-films, it is indeed reasonable to expect the local values of resistivity to have significant variations in time. Defect migration, nanoparticle coalescence, and the melting at different temperatures of nanoparticles of different size [40] are all mechanisms that can contribute to various degrees to those variations. In Ref. [14], a cyclic mechanism driven by the local flow of current, here recalled in Fig. 3, was proposed. Lacking of direct evidence and the support of a quantitative analysis, such a cycle is for now only hypothetical and in the following we shall discuss the modeling of a rather more general class of dynamical mechanisms. Nevertheless the cycle of Fig. 3 will be used throughout our discussion to show how our idea of modeling is carried out in practice.

For concreteness, we distinguish within the film regions that undergo some structural change, "active spots" and regions that do not, "inactive" ones. In the case of Ref. [14], an active spot is the boundary between two adjacent nanoparticles. The scale of the actual mechanism(s) may be larger,

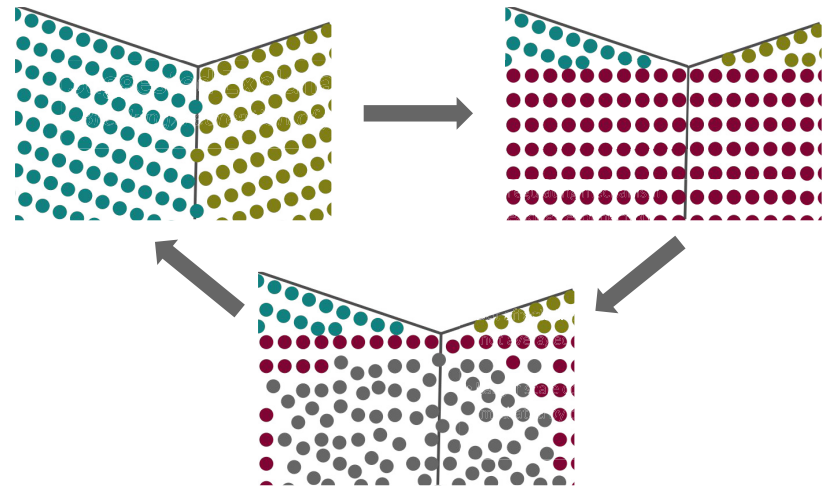

FIG. 3. A sketch of the hypothetical microscopic mechanism proposed in Ref. [14] to explain RS in ng-films. An active spot is identified with the interface between two nanoparticles (top left). Such a system makes a transition to a higher-conductivity state via defect migration (top right); from that, a lower-conducting state is reached because of local melting (bottom); finally, the original interface is rebuilt (top left) by action of the original crystals in the nanoparticles that do not participate to the transition (inactive spots, which surround the active ones).

and therefore an active spot can in fact comprehend various nanoparticles. Starting from a given initial atomic configuration, an active spot experiences over time a rearrangement of its atoms, as effect of temperature variations due to the current flowing through it. A rearrangement can either lead to a configuration with higher conductivity, if, for example, two nanoparticles coalesce or a defect migrates, or to a lower conductivity, as effect, for instance, of local melting. In time, several rearrangement may occur, and it is not to exclude the possibility that an active spot may be later found in its initial configuration, as seen in Fig. 3.

The presence of an onset time of a few seconds for the resistance jumps to start suggests that in this transient regime the spots are not active yet. We argue that a current is needed for the Joule heating to provide the missing energy to activate the spots. More specifically, we conceptualize the process sequence as follows: The activity of a spot is initiated as soon as the temperature of the spot exceeds a critical temperature $\bar{T}$ : When this situation is reached, the spot has sufficient energy to overcome the activation barrier that prevented the first rearrangement to occur. In fact, it is reasonable to expect that different kinds of structural rearrangement which an active spot may go through are affected in one way or another (i.e., activated or deactivated, at least to some degree) by the value of temperature at the spot. We therefore suppose that a few critical temperatures $\bar{T}_{1}, \bar{T}_{2}, \ldots$, which mark different energy thresholds that allow or forbid the relevant rearrangements, exist.

The actual evolution of an active spot is therefore determined by current-related fluctuations of the local temperature. In order to describe the overall dynamical evolution, we adopt a stochastic approach. More specifically, we consider a discrete time variable $t_{a}$, characterized by a step $\delta t=t_{a+1}-t_{a}$ that can be tuned to match the experimental sampling frequency (e.g., $\delta t=0.1 \mathrm{~s}$ in Ref. [13]). The dynamics of the system is determined by a rule that dictates how each single 
active spot changes its state from one time step to the next. We assume a Markovian evolution, according to which at each time step an active spot has a given probability of changing its state that only depends on its current state and the ng-film temperature. A complete characterization of the system is therefore achieved by specifying the corresponding transition probability matrix $P$. If, for instance, we assume a thermally regulated three-state cycle as in Ref. [14], then the transition probability matrix can be written as

$$
\begin{aligned}
P(T) & =\left(\begin{array}{lll}
\mathcal{P}_{1 \rightarrow 1} & \mathcal{P}_{1 \rightarrow 2} & \mathcal{P}_{1 \rightarrow 3} \\
\mathcal{P}_{2 \rightarrow 1} & \mathcal{P}_{2 \rightarrow 2} & \mathcal{P}_{2 \rightarrow 3} \\
\mathcal{P}_{3 \rightarrow 1} & \mathcal{P}_{3 \rightarrow 2} & \mathcal{P}_{3 \rightarrow 3}
\end{array}\right) \\
= & {\left[\begin{array}{ccc}
1-p_{12}(T) & p_{12}(T) & 0 \\
0 & 1-p_{23}(T) & p_{23}(T) \\
p_{31}(T) & 0 & 1-p_{31}(T)
\end{array}\right], }
\end{aligned}
$$

where the only three degrees of freedom of the matrix are parametrized by functions of the ng-film temperature $p_{\text {nm }}(T)$ for which $0 \leqslant p_{\text {nm }}(T) \leqslant 1$ and are taken to suddenly change around some critical temperatures $\bar{T}_{1}, \bar{T}_{2} \ldots$ Such a parametrization takes into account the fact that transitions can only happen in one direction (so, say, $\mathcal{P}_{1 \rightarrow 2}>0$ but $\mathcal{P}_{1 \rightarrow 3}=$ 0 ) and ensures that total probability $\mathcal{P}_{n \rightarrow 1}+\mathcal{P}_{n \rightarrow 2}+\mathcal{P}_{n \rightarrow 3}$ sums up to 1 . The functions $p_{\mathrm{nm}}(T)$ are characteristic of the microscopic mechanism one wants to model and their exact form can in principle be determined by means of numerical simulations of a single active spot. As they are function of the temperature, we must also specify how that quantity evolves with time.

Temperature variations of the ng-film happen for two reasons, namely (i) Joule heating, for which

$$
\Delta T m c=\Delta t V^{2} / R,
$$

$V$ being the applied voltage, $R$ the resistance of the ng-film, $m$ its mass and $c$ its specific heat, and (ii) heat dissipation due to contact with the environment, for which

$$
\Delta T=-\frac{\Delta t}{\tau}\left[T(t)-T_{\mathrm{env}}\right]
$$

with $\tau$ a characteristic time constant that describes how fast the film heat is transferred to the substrate and $T_{\text {env }}$ the environment temperature. Combining the two contributions into a law for discrete time propagation we can write

$$
T_{a+1}=T_{a}+\left[c_{1} \sigma_{e}\left(t_{a}\right)-c_{2}\left(T_{a}-T_{\mathrm{env}}\right)\right] \delta t,
$$

where $c_{1}$ and $c_{2}$ are two suitable constants and the film conductivity $\sigma\left(t_{a}\right)$ is calculated via a time-dependent version of Eq. (1), namely

$$
\sum_{i} \Phi_{i}\left(t_{a}\right) \frac{\sigma_{i}-\sigma_{e}\left(t_{a}\right)}{\sigma_{i}+2 \sigma_{e}\left(t_{a}\right)}=0,
$$

where the index $i$ runs over all the phases identified in the system while $a$ represents the time index. In the case of the mechanism of Fig. 3 we would introduce four phases: an insulating one for describing the voids, a polycrystalline characterized by a certain grain size (corresponding to the top
TABLE II. List of the parameters, except transition probabilities, used to obtain Fig. 4 and Fig. 5. While for the latter fully dimensional values were used, for the former the parameters are expressed in terms of three quantities (one for the conductivity, one for the temperature and the time step) that set the scale of the problem.

\begin{tabular}{lcc}
\hline \hline & Fig. 4 & Fig. 5 \\
\hline Length & & $1 \mathrm{~mm}$ \\
Width & & $1 \mathrm{~mm}$ \\
Thickness & 0 & $30 \mathrm{~nm}$ \\
$\sigma_{0}$ & & 0 \\
$\sigma_{1(0)}$ & $1450 \mathrm{~m}^{-1} \Omega^{-1}$ \\
$\alpha_{1}$ & 0 & $0.00013 \mathrm{~K}^{-1}$ \\
$\sigma_{2(0)}$ & $2175 \mathrm{~m}^{-1} \Omega^{-1}$ \\
$\alpha_{2}$ & $1.4 \sigma_{1(0)}$ & $0.00013 \mathrm{~K}^{-1}$ \\
$\sigma_{3(0)}$ & 0 & $725 \mathrm{~m}^{-1} \Omega^{-1}$ \\
$\alpha_{3}$ & $0.2 \sigma_{1(0)}$ & $0.00013 \mathrm{~K}^{-1}$ \\
Active spots & 0 & 2000 \\
Inactive spots & 200 & 0 \\
$\Phi_{0}$ & 0 & 0.504916 \\
$T_{0}$ & 0.3 & $300 \mathrm{~K}$ \\
$\bar{T}_{1}$ & $\left(1+10^{-5}\right) T_{0}$ & $710 \mathrm{~K}$ \\
$\bar{T}_{2}$ & $\left(1+2 \times 10^{-5}\right) T_{0}$ & $1392 \mathrm{~K}$ \\
$\bar{T}_{3}$ & $\left(1+3 \times 10^{-5}\right) T_{0}$ & $300 \mathrm{~K}$ \\
$T_{\text {env }}$ & $T_{0}$ & $0.005 \mathrm{~s}^{-1}$ \\
$c_{1}$ & $\left(6 \times 10^{6}\right)^{-1} T_{0}\left(\sigma_{1(0)} \delta t\right)^{-1}$ & $0.1 \mathrm{~s}^{-1}$ \\
$c_{2}$ & $10^{-3} \delta t^{-1}$ & \\
$\delta t$ & & \\
\hline \hline
\end{tabular}

left state), a second polycrystalline characterized by bigger grains (top right), and an amorphous one (bottom).

If the temperature of the sample spans a wide range of values, then the conductivity of the single phases may vary due to the increasing contribution of electron-phonon scattering events, in which case we must consider

$$
\sum_{i} \Phi_{i}\left(t_{a}\right) \frac{\sigma_{i}\left(T_{a}\right)-\sigma_{e}\left(t_{a}\right)}{\sigma_{i}\left(T_{a}\right)+2 \sigma_{e}\left(t_{a}\right)}=0
$$

instead of Eq. (7). Around room temperature, the resistivity of gold, from simple bulk [41] to very complex systems like nanocrystalline films [42], increases linearly with the temperature and therefore we consider $\sigma_{i}\left(T_{a}\right)=\sigma_{i(0)}\left[1+\alpha_{i}\left(T_{a}-\right.\right.$ $\left.\left.T_{\text {env }}\right)\right]^{-1}$, where $\alpha_{i}$ a positive parameter usually called temperature coefficient of resistivity (TRC). A signature of a nonvanishing TRC is a slight drift in an ohmic regime toward higher values of resistivity, which may be recognized in the resistance measurements of a ng-film under the bias of $0.5 \mathrm{~V}$ in Ref. [13].

Provided with numerical values for conductivity $\sigma_{i(0)}$ and the TRC $\alpha_{i}$ of the different phases, the parameters $c_{1}, c_{2}$ of the temperature equation (6), a specific behavior for the functions $p_{\mathrm{nm}}(T)$, the total number of spots and the phase initial densities, it is possible to propagate the model and simulate the time evolution of a ng-film. In Fig. 4 we present a specific realization of such a model, whose parameters, reported in Tables II and III, were chosen to give rise to the relevant features of the RS behavior observed in the experiments of Ref. [13]. We can indeed clearly distinguish a big spike and jumps between ohmic regimes characterized by different duration, fluctuation 

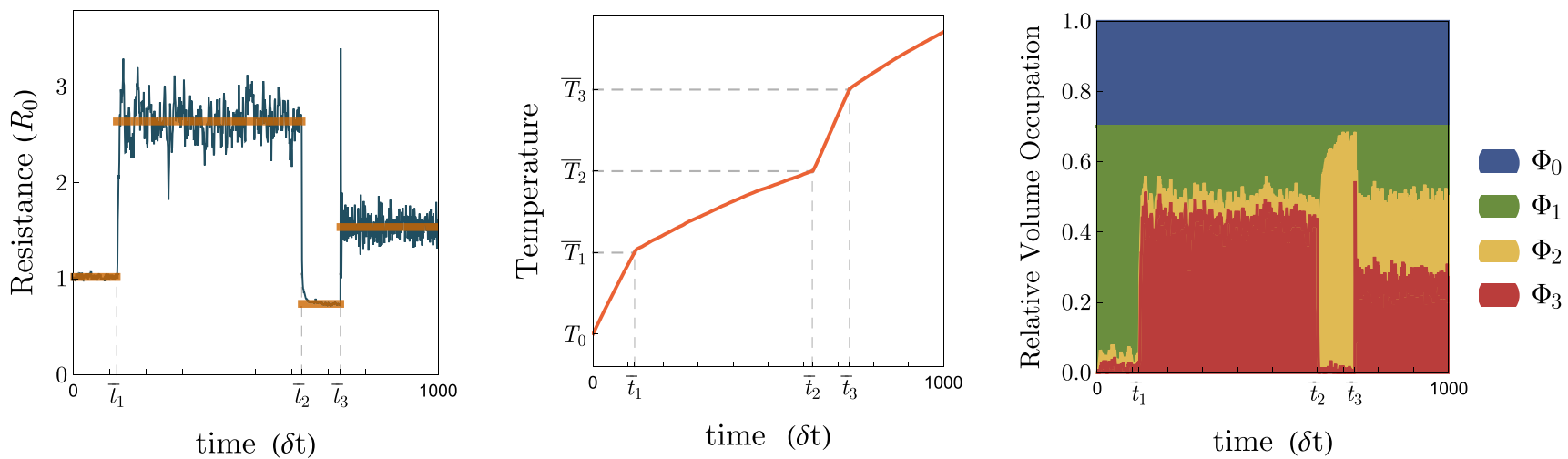

FIG. 4. Relative resistance (left), temperature (center), and relative volume occupied by the different phases (right) in a paradigmatic simulation of a ng-film with thermally regulated microscopic processes. A simple statistical analysis allows to calculate the average values of resistance (left, orange straight lines) without the need to run the simulation.

amplitude, and resistance value, which can be higher as well as lower than the initial value. The ohmic regimes are characterized by fluctuations due to the occurrence of the structural rearrangements of some active spots at each time step. The amplitude of the fluctuations reduces if the number of active spots is increased. A comparison between the temperature and resistance evolution shows that steps occur every time a critical temperature is crossed. In other words, whenever the transition probabilities are stable, only fluctuations occur, while jumps arise when transition probabilities suddenly change. The duration of a ohmic regime is therefore linked to the time the sample takes to reach a critical temperature. As in the experiments, a voltage threshold, under which no jumps but only fluctuations occur, is also observed, as result of the fact that Joule heating, which is proportional to $V^{2}$, is not sufficient to take the ng-film to cross the lowest critical temperature. Rising the voltage well above the threshold can significantly accelerate the dynamical evolution, as critical temperatures are reached in a shorter time.

Such a simulation therefore teaches us that jumps of resistivity, such as those occurring at $t_{1}, t_{2}$, and $t_{3}$ in Fig. 4 , are not caused by structural changes involving single active spots (e.g., the melting of a juction of the migration of a grain boundary). Far from the percolation threshold it would take much more than a single boundary to melt to give rise to a macroscopic jump. In the simulated sample, such microscopic events happen very frequently and in fact they are at the origin of the "noise" one can see during the periods of stability of the resistance. What causes the jumps is the sudden prevalence of one phase over the others, as shown in the last plot of Fig. 4. In other words, during the entire time of the measuring process, the relative concentration of the three phases depicted

TABLE III. Values of the functions that identify the transition probability matrix Eq. (2) for the realization of the model leading to Fig. 4.

\begin{tabular}{ccccc}
\hline \hline & $T<\bar{T}_{1}$ & $\bar{T}_{1}<T<\bar{T}_{2}$ & $\bar{T}_{2}<T<\bar{T}_{3}$ & $\bar{T}_{3}<T$ \\
\hline$p_{12}$ & 0.01 & 0.2 & 0.1 & 0.8 \\
$p_{23}$ & 0.3 & 0.8 & 0.008 & 0.8 \\
$p_{31}$ & 0.4 & 0.1 & 0.7 & 0.7 \\
\hline \hline
\end{tabular}

in Fig. 3 is mostly stable, but sometimes it changes quite abruptly. This is a consequence of the fact that the transitions of an active spot from one state to another are (de)activated at certain temperatures and, each time the sample crosses a critical temperature, a certain state becomes dominant over the others.

A statistical analysis allows to exactly calculate the values of resistivity spanned during the ohmic regimes starting from the transition probability matrix defined in Eq. (2) and the values of conductivity of the single phases. During such regimes, the temperature of the ng-film is between two critical temperatures and the transition probability matrix is constant, $P(T)=P$. We can then say [43] that the average concentration of the different phases $\langle\Phi\rangle_{i}$ is given by $\langle\Phi\rangle_{i}=P_{i 1}^{\infty}$, where $P^{\infty}$ is the limiting transition matrix $P^{\infty}=P P P, \ldots$, for which $P_{1 m}^{\infty}=P_{2 m}^{\infty}=P_{3 m}^{\infty}$. Provided with $\langle\Phi\rangle_{i}$ for each ohmic regime, one can use Eq. (1) to calculate the effective conductivity, and hence the resistance of the film, for the such regimes without performing a propagation of the stochastic equations. Such an analysis, whose numerical outcome for this specific case is reported in Fig. 4 on top of the stochastic simulation, is particularly important for cases in which the conditions of the simulation, or, for that matter, of an experiment, cannot ensure that the entire landscape of allowed values is spanned.

Finally, to prove that the approach can quantitatively, and not only qualitatively, explain the experimental data, in Fig. 5 we show another realization of the model, whose parameters were tuned to match the experimental results for the ng-film with thickness $30 \mathrm{~nm}$ under the bias of $0.5 \mathrm{~V}$, reported in Ref. [13], Fig. 4(c). In fact, since the same graph $R(t)$ can be obtained with various choices of the parameters, we assumed certain features of the spots that one would expect from the specific mechanisms represented in Fig. 3. More specifically, we assumed a certain ratio between the conductivity of the different phases and an overall behavior of the functions $p_{\mathrm{nm}}(T)$ characterized by two critical temperatures, one corresponding to the defect migration activation temperature and the other to the local melting temperature. Given then the specifics of the experiment (sample size and density, and frequency of the current measurements), we tuned the two parameters of the conductivity of the first phase $\sigma_{1(0)}$ and $\alpha_{1}$, which set a scale for the resistance, the constant $c_{2}$, which 


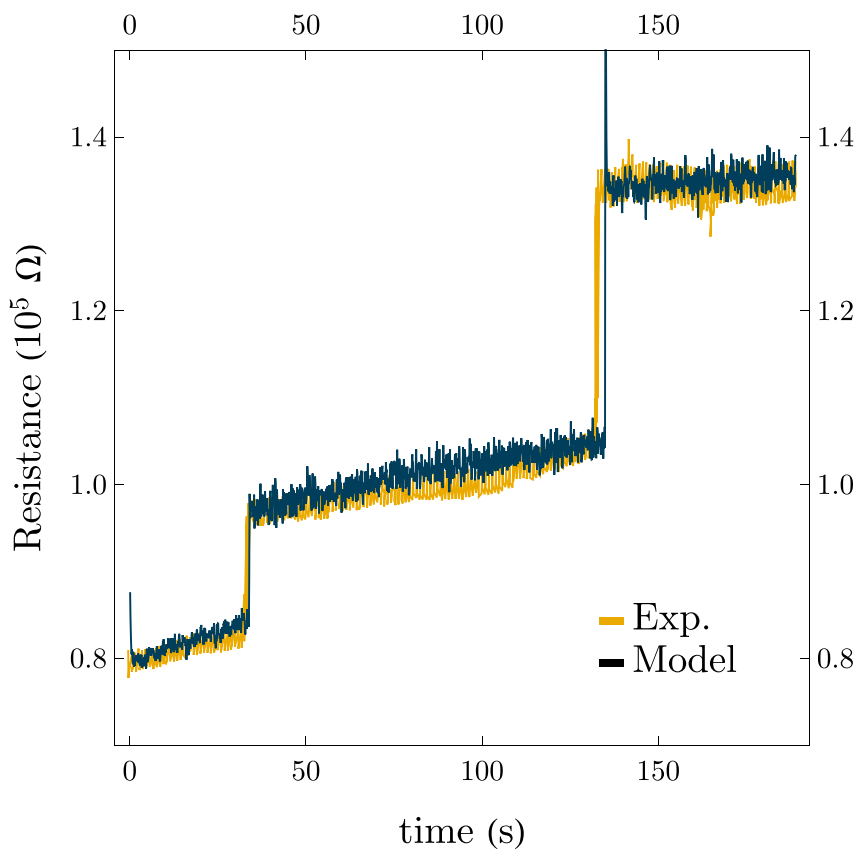

FIG. 5. Resistance of a simulated ng-film with microscopic parameters tailored to give rise to the RS reported in Ref. [13], Fig. 4(c).

determines the timescale of the problem, and the number of active spots, which sets the amplitude of the fluctuations in the ohmic regimes, to match the corresponding features of the experimental data (cf. Tables II and IV). Such a careful choice of the parameters allows to reproduce the experimental data with a sufficient level of accuracy. Such an accuracy, however, must not be mistaken with a proof that the modeled microscopic processes indeed occur and the values used for the parameters, although plausible, reflect their real values. The available information on the experimental setup is not enough to sufficiently restrict the space of parameters compatible with the experimental data. The same macroscopic resistance can be achieved with quite different choices of the parameters. In fact, also the details of the modeled microscopic mechanism, including even its cyclicity, are not necessary ingredients to reproduce the observed data; what is important is the presence of thermally (de)activated local structural rearrangements. The level of accuracy of the simulation therefore only supports the adequacy of the mathematical tools we here put together for describing this form RS we are interested in.

\section{CONCLUSIONS}

Nanogranular gold films with thickness well beyond the percolation threshold present an intriguing, unexpected

TABLE IV. Values of the functions that identify the transition probability matrix Eq. (2) for the realization of the model leading to Fig. 5.

\begin{tabular}{cccc}
\hline \hline & $T<\bar{T}_{1}$ & $\bar{T}_{1}<T<\bar{T}_{2}$ & $\bar{T}_{2}<T$ \\
\hline$p_{12}$ & 0.2 & 0.9 & 0.2 \\
$p_{23}$ & 0.1 & 0.3 & 0.8 \\
$p_{31}$ & 0.7 & 0.7 & 0.3 \\
\hline \hline
\end{tabular}

dynamical response to external electrostatic potentials. Understanding and, ultimately, harnessing such a phenomenon can lead to interesting technological applications. Their modeling, however, poses some theoretical challenges.

Even before introducing dynamical effects, estimating the resistivity of a nanogranular film eludes the standard models used for crystal films which do not take into account the complex structure arising from the presence of nanoparticles. In this work we suggest to approach the problem using Bruggeman's formalism for multicomponent media, which belongs to the framework of the effective medium approximations. The approach was designed for macroscopic scales [33] and is expected to be accurate only when a sufficiently large number of percolation paths have been established [10] (a regime that for inhomogeneous systems is not always easy to establish by simply looking at the overall resistance [44]). Apart from those caveats, which may be resolved by resorting to more sophisticated approximations [25], the method is typically rather accurate, computationally very inexpensive and, most importantly for our case, quite flexible. We have indeed explained how it can be adapted to describe not only the porosity but also other features of ng-films, such as amorphous layers at the nanoparticle interfaces and the presence of nanoparticles of different size, complementing other models designed to account for the remaining sources of RR.

Provided with such a tool it is possible to build dynamical models. To give a concrete example of the flexibility and extent of the approach, we built a stochastic model connecting the macroscopic film resistance to a generic class of microscopic mechanisms, characterized by local variations of resistivity due to structural rearrangements. Two specific realizations of the model, with tailored choice of the parameters, were shown to reproduce, qualitatively as well as quantitatively, the characteristic jumps of resistivity, as well as other, less obvious, features of the experimental data. Our simulations proved that, far from the percolation threshold, in a regime where local structural rearrangements are not sufficient to explain macroscopic jumps of resistance, RS can arise if those rearrangements are thermally (de)activated; as the sample temperature rises as effect of Joule heating, certain rearrangements become more favorable than others and when such a transition happen an appreciable jump of resistance occurs. Since local structural rearrangements happening in nanogranular films can be investigated by means of atomistic simulations that only have to involve a few nanoparticles, such a model can be considered as a starting point for further theoretical investigations aimed at shading light on the such mechanisms.

In summary, we presented a modeling based on EMA that can be used to study the RS phenomena that seems to characterize nanogranular film also beyond the percolation threshold and we showed how simple thermally regulated local structural changes can explain the most relevant features of the experiments, suggesting a clear path for future investigations.

\section{ACKNOWLEDGMENT}

This work was fully funded by Fondazione CON IL SUD (Grant No. 2018-PDR-01004). 
[1] Y. Chen, H. (H.) Li, C. Wu, C. Song, S. Li, C. Min, H.-P. Cheng, W. Wen, and X. Liu, Neuromorphic computing's yesterday, today, and tomorrow-An evolutional view, Integration 61, 49 (2018).

[2] S. H. Lee, X. Zhu, and W. D. Lu, Nanoscale resistive switching devices for memory and computing applications, Nano Res. 13, 1228 (2020).

[3] J. S. Lee, S. Lee, and T. W. Noh, Resistive switching phenomena: A review of statistical physics approaches, Appl. Phys. Rev. 2, 031303 (2015).

[4] Y. Yang and W. Lu, Nanoscale resistive switching devices: Mechanisms and modeling, Nanoscale 5, 10076 (2013).

[5] B. Hwang and J.-S. Lee, Recent advances in memory devices with hybrid materials, Adv. Electron. Mater. 5, 1800519 (2019).

[6] P. Borziak, V. Dyukov, A. Kostenko, Yu. Kulyupin, and S. Nepijko, Electrical conductivity in structurally inhomogeneous discontinuous metal films, Thin Solid Films 36, 21 (1976).

[7] A. Sattar, S. Fostner, and S. A. Brown, Quantized Conductance and Switching in Percolating Nanoparticle Films, Phys. Rev. Lett. 111, 136808 (2013).

[8] C. Minnai, A. Bellacicca, S. A. Brown, and P. Milani, Facile fabrication of complex networks of memristive devices, Sci. Rep. 7, 7955 (2017).

[9] S. Fostner and S. A. Brown, Neuromorphic behavior in percolating nanoparticle films, Phys. Rev. E 92, 052134 (2015).

[10] S. Kirkpatrick, Percolation and conduction, Rev. Mod. Phys. 45, 574 (1973)

[11] D. Stauffer and A. Aharony, Introduction to Percolation Theory (Taylor \& Francis, London, 1994).

[12] M. Sahimi, Applications of Percolation Theory (Taylor \& Francis, London, 1994).

[13] M. Mirigliano, F. Borghi, A. Podestà, A. Antidormi, L. Colombo, and P. Milani, Non-ohmic behavior and resistive switching of au cluster-assembled films beyond the percolation threshold, Nanosc. Adv. 1, 3119 (2019).

[14] M. Mirigliano, D. Decastri, A. Pullia, D. Dellasega, A. Casu, A. Falqui, and P. Milani, Complex electrical spiking activity in resistive switching nanostructured au two-terminal devices, Nanotechnology 31, 234001 (2020).

[15] A. Diaz-Alvarez, R. Higuchi, P. Sanz-Leon, I. Marcus, Y. Shingaya, A. Z. Stieg, J. K. Gimzewski, Z. Kuncic, and T. Nakayama, Emergent dynamics of neuromorphic nanowire networks, Sci. Rep. 9, 14920 (2019).

[16] S. Fostner, R. Brown, J. Carr, and S. A. Brown, Continuum percolation with tunneling, Phys. Rev. B 89, 075402 (2014).

[17] A. Loeffler, R. Zhu, J. Hochstetter, M. Li, K. Fu, A. Diaz-Alvarez, T. Nakayama, J. M. Shine, and Z. Kuncic, Topological properties of neuromorphic nanowire networks, Front. Neurosci. 14, 184 (2020).

[18] J. B. Mallinson, S. Shirai, S. K. Acharya, S. K. Bose, E. Galli, and S. A. Brown, Avalanches and criticality in self-organized nanoscale networks, Sci. Adv. 5, eaaw8438 (2019).

[19] H. G. Manning, F. Niosi, C. G. da Rocha, A. T. Bellew, C. O'Callaghan, S. Biswas, P. F. Flowers, B. J. Wiley, J. D. Holmes, M. S. Ferreira, and J. J. Boland, Emergence of winnertakes-all connectivity paths in random nanowire networks, Nat. Commun. 9, 3219 (2018).

[20] G. Milano, G. Pedretti, M. Fretto, L. Boarino, F. Benfenati, D. Ielmini, I. Valov, and C. Ricciardi, Brain-inspired structural plasticity through reweighting and rewiring in multi-terminal self-organizing memristive nanowire networks, Adv. Intell. Syst. 2, 2000096 (2020).

[21] M. D. Pike, S. K. Bose, J. B. Mallinson, S. K. Acharya, S. Shirai, E. Galli, S. J. Weddell, P. J. Bones, M. D. Arnold, and S. A. Brown, Atomic scale dynamics drive brain-like avalanches in percolating nanostructured networks, Nano Lett. 20, 3935 (2020).

[22] H. O. Sillin, R. Aguilera, H.-H. Shieh, A. V. Avizienis, M. Aono, A. Z. Stieg, and J. K. Gimzewski, A theoretical and experimental study of neuromorphic atomic switch networks for reservoir computing, Nanotechnology 24, 384004 (2013).

[23] R. Landauer, Electrical conductivity in inhomogeneous media, in Electrical Transport and Optical Properties of Inhomogeneous Media, edited by J. C. Garland and D. B. Tanner, AIP Conf. Proc. No. 40 (AIP, New York, 1978), p. 2.

[24] D. Stroud, The effective medium approximations: Some recent developments, Superlatt. Microstruct. 23, 567 (1998).

[25] C. Grimaldi, Theory of percolation and tunneling regimes in nanogranular metal films, Phys. Rev. B 89, 214201 (2014).

[26] A. F. Hebard and S. B. Arnason, Bad-metal behavior: Exotic physics or a consequence of microstructure? J. Supercond. 12, 159 (1999).

[27] R. D. Gould, S. Kasap, and A. K. Ray, Thin films, in Springer Handbook of Electronic and Photonic Materials, edited by Safa Kasap and Peter Capper (Springer International Publishing, Cham, 2017), pp. 1-1.

[28] A. F. Mayadas and M. Shatzkes, Electrical-resistivity model for polycrystalline films: The case of arbitrary reflection at external surfaces, Phys. Rev. B 1, 1382 (1970).

[29] K. Fuchs, The conductivity of thin metallic films according to the electron theory of metals, Math. Proc. Cambr. Philos. Soc. 34, 100 (1938).

[30] E. H. Sondheimer, The mean free path of electrons in metals, Adv. Phys. 1, 1 (1952).

[31] G. Benetti, C. Caddeo, C. Melis, G. Ferrini, C. Giannetti, N. Winckelmans, S. Bals, M. J. Van Bael, E. Cavaliere, L. Gavioli, and F. Banfi, Bottom-up mechanical nanometrology of granular ag nanoparticles thin films, J. Phys. Chem. C 121, 22434 (2017).

[32] Y. Namba, Resistivity and temperature coefficient of thin metal films with rough surface, Jpn. J. Appl. Phys. 9, 1326 (1970).

[33] D. J. Bergman and D. Stroud, Physical Properties of Macroscopically Inhomogeneous Media, (Academic Press, San Diego, CA, 1992), pp. 147-269.

[34] T. C. Choy, Effective Medium Theory: Principles and Applications, International Series of Monographs on Physics (Oxford University Press, Oxford, 2015).

[35] J. M. Luck, Conductivity of random resistor networks: An investigation of the accuracy of the effective-medium approximation, Phys. Rev. B 43, 3933 (1991).

[36] D. A. G. Bruggeman, Berechnung verschiedener physikalischer konstanten von heterogenen substanzen. i. dielektrizitätskonstanten und leitfähigkeiten der mischkörper aus isotropen substanzen, Ann. Phys. 416, 636 (1935).

[37] R. A. Serway and J. R. Gordon, Principles of Physics, Principles of Physics, Vol. 2 (Saunders College, Philadelphia, PA, 1998).

[38] K. K. Mohanty, Porous media: Fluid transport and pore structure, AIChE J. 38, 1303 (1992).

[39] R. Henriquez, M. Flores, L. Moraga, G. Kremer, C. GonzálezFuentes, and R. C. Munoz, Electron scattering at surfaces and 
grain boundaries in thin au films, Appl. Surf. Sci. 273, 315 (2013).

[40] J. E. Morris, Nanoparticle properties, in Nanopackaging, edited by J. E. Morris (Springer, New York, 2008), 1st ed., chap. 5, pp. 93-107.

[41] D. C. Giancoli, Physics: Principles with Applications, 6th ed. (Pearson Education, Upper Saddle River, NJ, 2005).

[42] J. Ederth, L. B. Kish, E. Olsson, and C. G. Granqvist, Temperature dependent electrical resistivity in nanocrystalline gold films made by advanced gas deposition, J. Appl. Phys. 88, 6578 (2000).

[43] W. J. Stewart, Probability, Markov Chains, Queues, and Simulation: The Mathematical Basis of Performance Modeling (Princeton University Press, Princeton, NJ, 2009).

[44] M. Creyssels, C. Laroche, E. Falcon, and B. Castaing, Pressure dependence of the electrical transport in granular materials, Eur. Phys. J. E 40, 56 (2017). 